\title{
Original
}

\section{Las miasis como entidad de interés en Medicina del Trabajo}

\section{Myiasis as an entity of interest in occupational medicine}

\author{
Alejandro González Medina ', Francisco Archilla Peña ${ }^{2}$, Gilberto Jiménez Ríos ${ }^{1}$ \\ 1. Instituto de Medicina Legal de Granada. España. \\ 2. Departamento de Anatomía y Embriología Humana, Universidad de Granada. España.
}

Recibido: 20-09-11

Aceptado: 30-09-11

\author{
Correspondencia \\ Alejandro González Medina \\ Instituto de Medicina Legal de Granada \\ Avenida de Madrid, s/n \\ 18012 Granada. España. \\ Tfno.: 958204231 \\ E-mail: agm@ugr.es
}

ResUmen

En escasas ocasiones se ha hecho referencia a las miasis en el ámbito de la Medicina del Trabajo. Es un error bastante generalizado pensar que este tipo de parasitación es exclusiva de países subdesarrollados o de estratos sociales carentes de las mínimas medidas de higiene. Sin embargo, es importante conocer los agentes etiológicos y advertir del riesgo que afecta a ciertos sectores laborales expuestos a materia orgánica en descomposición o a ciertos alimentos que por sus características químicas actúan de atrayentes para los dípteros. Para ello, estudiamos los casos de miasis que se diagnosticaron en varios centros sanitarios ubicados en Granada, Málaga, Almería y Jaén durante el periodo comprendido entre septiembre de 2010 y junio de 2011 y consideramos con especial interés los que se iniciaron por una infestación durante el periodo laboral. Se observa en las miasis laborales un predominio de las especies Piophila casei (Linnaeus, 1758) y Sarcophaga (Bercaea) africa (Wiedemann, 1824). Asimismo, exponemos las principales características del ciclo vital de los dípteros de interés sanitario y las medidas de seguridad que deben adoptarse para evitar este tipo de accidente laboral.

Med Segur Trab (Internet) 2011; 57 (225) 331-338

Palabras clave: Miasis; Parasitología; Díptero; Larva; Entomología Forense.

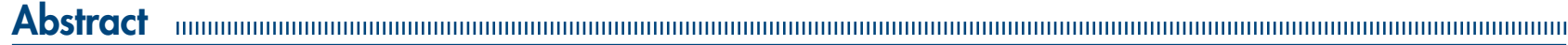

In few occasions, reference to myiasis in the scope of Occupational Medicine has been done. It is a generalized mistake to think that this type of parasitation is exclusive of underdeveloped countries or social layers without the minimal hygiene measures. Nevertheless, it is important to know the etiological agents and to notice the risk that affects certain occupational sectors exposed to organic matter in decay proccess or to certain foods that by their chemical characteristics act of appealing to the Diptera. We studied the cases of myiasis diagnosed in some medical centers located in Granada, Málaga, Almería and Jaén during the period between September 2010 and June 2011 and we considered with special interest those that began by an infestation during the working period. A predominance of Piopbila casei (Linnaeus, 1758) and Sarcophaga (Bercaea) africa (Wiedemann, 1824) was observed in laboral myiasis. Also we exhibit the main characteristics
\end{abstract}


of the vital cycle of Diptera of sanitary interest and the safety measures that must be adopted to avoid this type of occupational accident.

Med Segur Trab (Internet) 2011; 57 (225) 331-338

Key words: Myiasis; Parasitology; Diptera; Larvae; Forensic Entomology. 


\section{INTRODUCCIÓN}

Se entiende por "miasis" la infestación de seres vivos por parte de los estadios inmaduros de dípteros para completar su ciclo vital y alimentarse durante un periodo de tiempo variable de sus tejidos, exudaciones o alimentos ingeridos (1). Suele ser un problema que, con frecuencia, presenta un carácter de índole veterinario ya que el acceso al hospedador se encuentra facilitado por la estabulación, los contenedores de alimento y la naturaleza de estos o la falta de mecanismos eficientes a la hora de repeler la colonización de los dípteros. La mejora en la calidad de vida y las medidas de higiene frente a los restos orgánicos en descomposición (basuras, cadáveres...) hizo postular a algunos especialistas que estas enfermedades quedarían restringidas al ámbito tropical, a los países del Tercer Mundo o a aquellas personas que por motivos culturales o económicos estuviesen en contacto con desechos orgánicos ${ }^{2}$. Mientras que en poblaciones humanas con estas características se han desarrollado sistemas de prevención y saneamiento de mayor o menor eficacia, el resto del mundo ha asumido la presencia ocasional de moscas en diferentes medios como algo normal que no merece mayor precaución. Solamente en el contexto de la industria alimentaria ha considerado el potencial patogénico de las moscas como transportadores de bacterias como Salmonella, Escherichia coli ${ }^{3}$ o de aminas putrefactivas debidas a la previa colonización de otros medios alimenticios o materias descompuestas (conocidas clásicamente como ptomaínas) que las generan ${ }^{4}$.

No obstante, los Dípteros no sólo tienen un papel de hospedador intermediario pasivo sino que ellos mismos pueden ser agentes parasitarios activos en ambientes integrados en sociedades altamente tecnológicas ${ }^{5}$. La gestión de residuos tanto urbanos como ganaderos o agrícolas, el contacto con cadáveres animales y humanos y el contacto con animales sensibles a esta modalidad de parasitación hacen que los seres humanos entremos en contacto con el principal sustrato alimenticio de los dípteros y que, en la mayoría de casos de forma accidental, la salud del trabajador corra un riesgo que podría evitarse.

En este artículo, estudiamos varios casos de miasis profesionales dentro del contexto de esta patología en varias provincias de Andalucía durante el periodo de diez meses. Ya que no se trata de una patología frecuente, son necesarios estudios de mayor escala temporal para caracterizar con mayor precisión esta entidad patológica.

\section{MATERIAL Y MÉTODOS}

La recogida de material entomológico fue llevada a cabo por los médicos internistas de seis centros hospitalarios en la provincia de Granada, cuatro en la de Málaga, tres en la de Jaén y el mismo número en la de Almería. Ante la ausencia de protocolos estandarizados de extracción y procesamiento de muestras entomológicas en casos de miasis, revisamos las técnicas propuestas en diferentes casos clínicos publicados en revistas especializadas, por ejemplo ${ }^{6-8}$. En muchas de ellas, se propone la utilización de alguna sustancia aislante (parafina, cera, derivados de petróleo...) para provocar la asfixia de las larvas antes de proceder a la extracción con pinzas, con el fin de reducir la fuerza de agarre a los tejidos infestados ${ }^{9}$. No obstante, se ha podido comprobar en ganado y animales domésticos que esta medida pre-extracción no es necesaria en las miasis facultativas o accidentales, ya que la fuerza de agarre de las larvas al medio no es especialmente fuerte. Para seguir un protocolo normalizado y aceptado por la comunidad científica, hemos elegido las normas y recomendaciones de la European Association for Forensic Entomology (EAFE) ${ }^{10}$. Aunque es un protocolo enfocado a la recogida de evidencias de interés en Entomología Forense, la naturaleza común de las evidencias muestreadas hace que la aplicación pueda ser común.

Una vez localizadas las larvas de dípteros en el cuerpo del paciente, se procede a extraerlas mediante el uso de pinzas blandas. Una parte de esas larvas se sumergen en agua hirviendo durante un minuto y posteriormente se conservan en alcohol etílico al 
$70 \% \mathrm{~m} / \mathrm{v}$. La otra parte de la muestra se conserva con sustrato alimenticio hasta que se pueden criar los estadios inmaduros a temperatura y humedad constantes en una incubadora Heraeus B12®. La cría controlada obedece a dos necesidades: la identificación de larvas en estadios demasiado tempranos (anteriores a larva III) puede arrojar bastantes errores a pesar de las claves diseñadas con esta intención ${ }^{11}$ y la data del momento de la infestación, con finalidades judiciales siempre que el caso lo requiera, siguiendo el mismo procedimiento que en la determinación del intervalo postmortem mínimo ${ }^{12}$. Una vez que se tienen larvas en estadio III o adultos, se procede a la identificación mediante el uso de claves dicotómicas específicas ${ }^{13,14,15}$.

La totalidad de los internistas que participaron en el estudio fueron instruidos antes del estudio mediante una sesión informativa y la difusión de artículos relacionados. Una vez recogidos los estadios inmaduros de los dípteros, uno de los autores los recogía en el centro de salud para su cría e identificación siempre que fuese posible.

En nuestro análisis hemos querido separar las miasis por exposición fuera del periodo laboral de aquellas que fueron contraídas durante el desempeño de la actividad laboral.

\section{RESULTADOS}

Tras un periodo de diez meses (entre septiembre de 2010 y junio de 2011), se recopiló la información sobre todos los casos atendidos (tabla I). La gran mayoría de las larvas pudo identificarse a partir de larva III, pero en el caso de los Sarcophagidae ha sido necesaria la cría hasta el estadio adulto.

Tabla I. Casos de miasis registrados en 16 centros de salud de las provincias de Granada, Málaga, Almería y Jaén durante un periodo de 10 meses

\begin{tabular}{cccccc}
\hline Provincia & $\begin{array}{c}\text { Código } \\
\text { de centro }\end{array}$ & Familia & Especie & Breve descripción del paciente & Tipo de miasis \\
\hline GRANADA & G-1 & Piophilidae & Piophila casei (Linnaeus, 1758) & $\begin{array}{c}\text { Manipulador de productos } \\
\text { cárnicos (jamón) }\end{array}$ & Ocular \\
& G-4 & Sarcophagidae & Sarcophaga (Bercaea) africa & Veterinaria de cooperativa & Traumática \\
& M-3 & Sarcophagidae & Sarcophaga (Bercaea) africa & Servicio de limpieza municipal & Ocular \\
MÁLAGA & A-1 & Caliphoridae & Lucilia caesar (Linnaeus, 1758) & Mendigo & Traumática \\
ALMERÍA & A-1 & Piophilidae & Piophila casei & Veterinaria de cooperativa & Traumática \\
& - & - & - & ganadera & -
\end{tabular}

Consideramos aparte las miasis producidas en un entorno profesional. Se puede deducir a partir de los entornos profesionales en los que se han producido las miasis que hay una serie de denominadores comunes que permiten englobar las profesiones de riesgo dentro de aquellas que están expuestas a:

- Materia orgánica en descomposición.

- Ganado estabulado o semiestabulado.

- Alimentos ricos en proteínas y lípidos que necesitan de un periodo de curación antes de entrar en la cadena de distribución.

En el caso del trabajador del servicio de limpieza, notificó a su médico que, cuando se encontraba limpiando un piso en el que el inquilino falleció y mostraba todos los síntomas de un síndrome de Diógenes, notó como algo "se le metió en el ojo" y tras frotarse desapareció momentáneamente el picor. Llevaba mascarilla, pero no protección ocular. 
Los veterinarios que se mencionan en la tabla I estaban realizando labores de retirada de cadáveres de reses muertas. Presentaban heridas de escasa profundidad en los brazos debidas a la manipulación habitual de reses estabuladas. Debido a que tal labor la desempeñaron en meses de primavera-verano, vestían con manga corta y, a pesar de llevar otras medidas de protección (gafas y mascarillas) las lesiones no estaban cubiertas.

Se describe también un caso en el que un trabajador que supervisaba el salado de jamones se acercó a comprobar a calidad de uno de los productos que, a su juicio, "presentaba pequeños gusanos que se movían y saltaban". Refirió con palabras similares al trabajador del servicio de limpieza el momento de la infestación pero no fue al médico porque pensó que se "trataba del polvo".

Como se puede observar, en la muestra correspondiente a las miasis profesionales predominan claramente las familias Sarcophagidae y Piophilidae con las especies Piophila casei (Linnaeus, 1758) (figura 1) y Sarcophaga (Bercaea) africa (Wiedemann, 1824) (figura 2).

Figura 1. Imagen a microscopio electrónico de barrido del extremo caudal de una larva en estadio III migratorio de Piophila casei del código de centro A. 1 a 350 aumentos

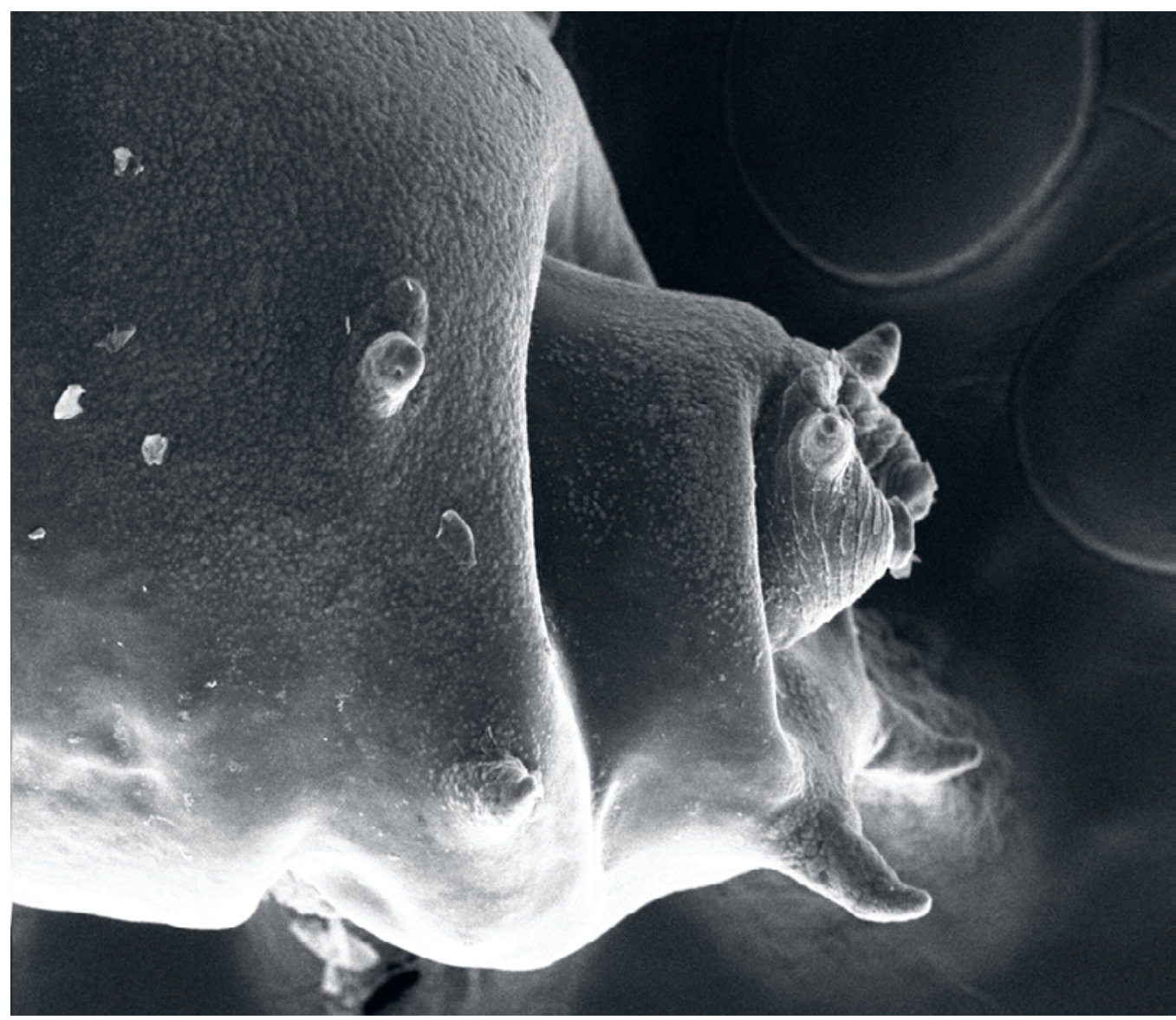


Figura 2. Genitalia masculina de Sarcophaga (Bercaea) africa. Pertenece a una de las larvas obtenidas del paciente correspondiente al centro sanitario con el código G.4 tras la cría en condiciones controladas

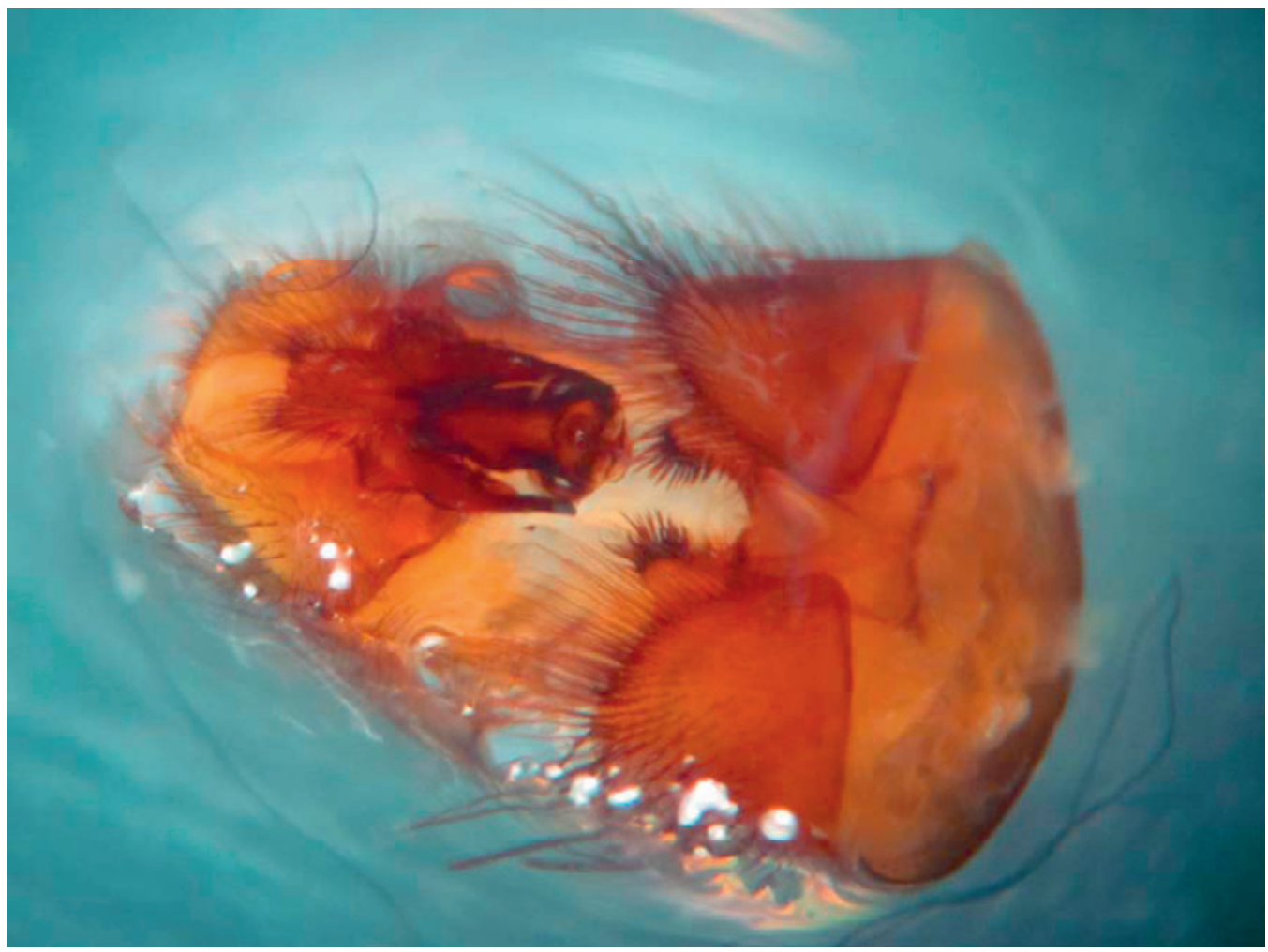

\section{DISCUSIÓN}

La Medicina del Trabajo ha sido desde sus inicios una disciplina que se ha caracterizado por el carácter multidisciplinario del cuerpo de conocimientos científicos que la conforman. Traumatólogos, patólogos forenses, oftalmólogos, toxicólogos y un largo etcétera han contribuido con los resultados de sus investigaciones a aumentar su corpus materiae. Sin embargo, la Entomología (y, concretamente, la Entomología Forense al abordar su aspecto más médico-legal) han permanecido al margen a pesar de su innegable carácter probatorio y preventivo ${ }^{16}$.

Los insectos eusinantrópicos (entendiendo por tales los que se encuentran en íntima relación con el entorno ocupado por el ser humano) ${ }^{17}$ son una realidad muchas veces ignorada por la mayoría de los trabajadores. La mayoría suelen ser conscientes del potencial patogénico que representan los adultos y las larvas de los dípteros como transmisores pasivos de enfermedades infecto-contagiosas. No obstante, el conocimiento del ciclo vital y de la biología de estos artrópodos podría facilitar la toma de decisiones preventivas de cara a un proceso parasitario. Estas medidas toman un carácter urgente en profesionales expuestos a los sustratos de los estadios inmaduros de insectos miasígenos.

La familia Calliphoridae es quizá una de las mejor conocidas por los entomólogos por ser la mayoría de sus especies los colonizadores primarios de restos orgánicos en descomposición ${ }^{18}$. En la sudeste de la Península Ibérica, encontramos una predominancia del género Calliphora en invierno y de los géneros Lucilia y Chrysomya en las estaciones más cálidas ${ }^{19}$. Su ciclo vital es prototípico dentro del orden Diptera. Tras ovopositar, las larvas pasan por tres estadios (el último de los cuales puede dividirse en una fase que todavía se alimenta y otra que se aleja de la fuente de alimentación), una fase de pupa y una posterior eclosión y salida del adulto, abandonando el pupario vacío ${ }^{20}$. Este ciclo, 
mientras no se diga lo contrario, es el que se cumplirá en las familias que se describen a continuación. Las larvas siguen un sistema de locomoción reptante (carecen de patas), de modo que sólo el contacto directo y sin protección puede originar una miasis facultativa.

Los Piophilidae son una familia de Dípteros que consta de 73 especies a nivel mundial, una docena de las cuales se pueden encontrar en la Península Ibérica ${ }^{21}$. Suelen ser atraídos por alimentos almacenados con alto contenido proteico y graso en donde depositan sus huevos ${ }^{22,23}$. Esto es compatible con el comportamiento necrófago de sus larvas en las últimas fases de descomposición de los cadáveres, cuando el contenido en sustancias volátiles rancias es liberado al exterior ${ }^{24}$. El rasgo más característico de las larvas de esta familia es la capacidad para plegarse sobre sí mismas y saltar en vertical, de ahí que reciban vulgarmente el nombre de "saltones" ${ }^{25}$. Este comportamiento se debe tener en cuenta a la hora de manipular posibles fuentes de alimento de estas larvas, ya que acercar demasiado la cabeza sin unas medidas de seguridad mínimas (mascarillas y gafas de protección) puede derivar en una miasis accidental. Las especies más frecuentemente asociadas a la contaminación de productos almacenados son Piophila casei y Stearibia nigriceps (Meigen, 1826), aunque esta última no se ha descrito todavía como causante de miasis.

Una particularidad que posee el ciclo reproductivo de los integrantes de la familia Sarcophagidae es su capacidad de larvipositar directamente sobre el sustrato alimenticio, ya que pueden retener en su cuerpo los huevos ya eclosionados ${ }^{26}$. Muchas veces, no es ni siquiera necesario que detengan el vuelo ya que una contracción brusca de parte de la musculatura abdominal puede hacer que las hembras proyecten las larvas hacia el exterior con cierta velocidad. Si existe una herida o un orificio corporal desprotegido, se puede desencadernar una miasis facultativa ya que las larvas de muchos Sarcophagidae tienen estructuras anatómicas adaptadas para una posible vida parasitaria. Incluso algunos integrantes de la familia son parásitos obligados, como el género Woblfabrtia y, en especial, la especie Woblfahrtia magnifica (Schiner, 1862) en el sur peninsular ${ }^{27}$.

Las familias mencionadas no son las únicas responsables de miasis. Aunque no se han podido obtener de muestras clínicas en nuestro estudio, se sabe que diferentes especies de otras familias de dípteros como los Phoridae, Gasterophilidae, Oestridae, Psychodidae y otras pueden ser responsables de miasis con variadas localizaciones ${ }^{1}$.

Por las circunstancias de las miasis estudiadas, hay ciertos sectores profesionales que no deben ignorarse de cara a la prevención. Durante el transcurso de ciertas autopsias, se ha descrito el comportamiento de salto por parte de miembros de la familia Piophilidae, lo que constituye un riesgo para los médicos forenses y auxiliares de autopsia que realizan el procedimiento. De igual forma, el queso y el yogur son alimentos cuya composición en proteínas e hidratos de carbono los hace idóneos para la colonización por parte de los Piophilidae. Sería recomendable que todos los trabajadores que se encuentren expuestos a estos sustratos presentaran una triple protección de mascarilla, gafas y vendajes que cubran las heridas que puedan quedar expuestas, por pequeñas que éstas sean.

La simple identificación de los estadios inmaduros o de los adultos criados en condiciones controladas muchas veces no es suficiente. En ocasiones, la intervención del entomólogo forense puede servir para datar la infestación de igual modo que se practica la data del intervalo post mortem mínimo en cadáveres. Esta datación tiene un importante valor legal a la hora de determinar posibles responsabilidades civiles o penales, pues puede originarse la duda del momento de la infestación y de si ésta se produjo en el ambiente laboral o fuera de él. La presencia de miasis en una persona física y mentalmente sana debe ser examinada con suma cautela para poder encuadrarla dentro de un lugar de trabajo quizá carente de ciertas medidas de seguridad a nuestro juicio imprescindibles para la salud de los trabajadores. 


\section{REFERENCIAS BIBLIOGRÁFICAS}

1. Zumpt F. Myiasis in Man and Animals in the Old World. London: Butterworths. 1965.

2. Geelhoed GW. Surgery and healing in the developing world. Texas: Landes Bioscience. 2005.

3. Fotedar R, Banerjee U, Singh S, Verma AK. The house fly (Musca domestica) as a carrier of pathogenic microorganisms in a hospital environment. J Hosp Infect. 1992; 20: 209 - 215.

4. Dekeirsschieter J, Verheggen FJ, Gohy M, Hubrecht F, Bourguignon L, Lognay G, Haubruge. Cadaveric volatile organic compounds released by decaying pig carcasses (Sus domesticus L.) in different biotopes. Forensic Sci Int, 2009; 189(1-3): 46 - 53

5. Jain A, Desal RU, Ehrlich J. Fulminant orbital myiasis in the developed world. Br J Ophtalmol. 2007; 91: 1565 - 1566.

6. Romero-Cabello R, Sánchez-Vega JT, Tay-Zavala J, Ruiz-Sánchez D, Calderón-Romero L. Miasis asociada a síndrome de complejo vascular periférico. Parasitol Latimoam. 2004; 59 (3-4): 159 - 161.

7. Yewhalaw D, Legesse W, Gebre-Selassie S, Kloos H. Human myiasis in an endemic area of Southwestern Ethiopia: Prevalence, knowledge, perceptions and practices. Ethiop J Health Dev. 2007; $21(2): 166$ - 172.

8. Gursel M, Aldemir OS, Ozgur Z, Ataoglu T. A rare case of gingival myiasis caused by diptera (Calliphoridae). J Clin Periodontol, 2002; 29: 777 - 780.

9. Caissie R, Beaulieu F, Giroux M, Berthod F, Landry PE. Cutaneous myiasis: diagnosis, treatment and prevention. J Oral Maxillofac Surg, 2008; 66: 560-568.

10. Amendt J, Campobasso CP, Gaudry E, Reiter C, LeBlanc HN, Hall MJR. Best practice in forensic entomology - standards and guidelines. Int J Legal Med, 2007; 121: 90 - 104.

11. Szpila K, Villet M. Morphology and identification of first instar larvae of African blowflies (Diptera: Calliphoridae) commonly of forensic importance. Journal of Medical Entomology, 2011; 48(4): 738 - 752.

12. Greenberg B. Flies as forensic indicators. J Med Entomol, 1991; 28(5): 565 - 577.

13. Szpila K. Key for the identification of third instars of European blowlfies (Diptera: Calliphoridae) of forensic importance. En: Current concepts in forensic entomology. 2010. Dordrecht-Heidelberg-LondonNew York. Springer. 43-56.

14. Velásquez Y, Magaña C, Martínez-Sánchez A, Rojo S. Diptera of forensic importance in the Iberian Peninsula: larval identification key. Med Vet Entomol, 2010; 24: 293 - 308.

15. Pape T. The Sarcophagidae (Diptera) of Fennoscandia and Denmark. Leiden - Copenhagen: E. J. Brill/ Scandinavian Science Press. 1987.

16. Catts EP, Goff ML. Forensic entomology in criminal investigations. Ann Rev Entomol, 1992; $37: 253$ - 272.

17. Gregor F, Povolny D. Versuch einer Klassifikation der Synanthropen Fliegen. J Hyg Epidemiol Microbiol Immunol. 1958, 2: 205-216.

18. Martínez-Sánchez A, Rojo S, Marcos-García MA. Annual and spatial activity of dung flies and carrion in a Mediterranean holm-oak pasture ecosystem. Med Vet Entomol, 2000; 14: 56 - 63.

19. Arnaldos MI, Romera E, Presa JJ, Luna A. Studies on seasonal arthropod succession on carrion in the southeastern Iberian Peninsula. Int J Legal Med, 2004; 118: 197-205.

20. Danks HV. Measuring and reporting life-cycle duration in insects and arachnids. Eur J Entomol, 2000; 97: $285-303$.

21. McAlpine JF. A revised classification of the Piophilidae including "Neottiophilidae" and "Thyreophoridae" (Diptera: Schizophora). Mem Entomol Soc Can, 1977; 103: 1 - 66.

22. Zuska J, Laštovka P. A review of the Czechoslovak species of the family Piophilidae with special reference to their importance to food industry (Diptera, Acalyptrata). Acta Entomol Bohemoslov, 1965; 62: $141-157$

23. Kirinoki M, Hitosugi M, Chigusa Y, Kurosu A, Tokudome S. Larvae of the family Piophilidae found in the marrow space of skeletal remains during a forensic autopsy. Med Entomol Zool, 2010; 61(2): 115 - 119.

24. Kumara TK, Abu Hassan A, Che Salmah MR, Bhupinder S. The infestation of Dermestes ater (De Geer) on a human corpse in Malaysia. Trop Biomed, 2009; 26: 73 - 79.

25. Bonduriansky F. Leaping behaviour and response to moisture and sound in larvae of piophilid carrion flies. Can Entomol, 2002; 134: 647 - 656.

26. Hall RD, Doisy KE. Length of time after death: effect on attraction and oviposition or larviposition of midsummer blow flies (Diptera: Calliphoridae) and flesh flies (Diptera: Sarcophagidae) of medicolegal importance in Missouri. Ann Entomol Soc Am, 1993; 86: 589 - 593.

27. Ruíz Martínez IR, Leclercq M. Data on distribution of screwworm fly Woblfabrtia magnifica (Schiner) in Southwestern Europe (Diptera: Sarcophagidae). Notes Fauniques de Gembloux, 1994; 28: 53-60. 